Military Technical College Kobry El-Kobbah, Cairo, Egypt

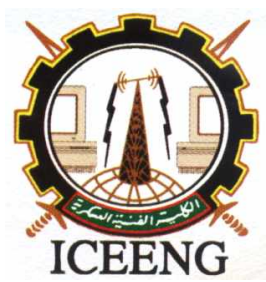

\author{
$6^{\text {th }}$ International Conference \\ on Electrical Engineering \\ ICEENG 2008
}

\title{
Performance analysis of MC DS-CDMA with generalized selection combining over land mobile satellite communication channels
}

By

Salah El-Agooz*,

\author{
Gamal Mabrouk Abdel-Hamid* \\ dein*
}

S. Shams El-

\section{ABSTRACT}

Two classes of multicarrier direct sequence code-division multiple-access are defined, namely Multi-tone DS-CDMA and Orthogonal multicarrier DS-CDMA. Their performances are considered over land mobile satellite communication channel with $\mathrm{M}$ branches of total $\mathrm{L}$ branches available diversity using generalized selection combining technique GSC(M,L). The average bit error rate for the two classes are investigated and compared using coherent binary phase shift keying modulation. It is assumed that the channel consists of Nakagami distributed shadowed line-of-sight signal plus Rayleigh distributed multipath signals. Using measured channel parameters, the performance is evaluated for light, average and heavy shadowing and fading environments.

Key words:

Orthogonal MC DS-CDMA, Multitone DS-CDMA, Land Mobile Satellite Channel (LMSC), Generalized selection Combining (GSC(M,L)).

* Military Technical College, Kobry El-Koppa, Cairo, Egypt 


\section{Introduction}

The land-mobile satellite systems seem to be the solution for realizing the global personal communication networks. They can be provide a wide range of services, recent investigations show that direct sequence code division multiple access (DS-CDMA) allows higher system capacity than any other multiple access technique [1].

In the context of spread-spectrum communication with RAKE reception, the complexity of MRC and EGC receivers depends on the number of resolvable paths available, which can be quite high, especially for multipath diversity of wide-band spread spectrum signals. In addition, MRC is sensitive to channel estimation errors, and these errors tend to be more important when the instantaneous SNR is low. On the other hand, SDC and SSC (switch and stay combining) use only one path out of the L resolvable paths available and hence do not fully exploit the amount of diversity offered by the channel.

Recently, there has been an interest in bridging the gap between these two extremes (MRC/EGC and SDC) by proposing generalized selection combining (GSC), which adaptively combines the $\mathrm{M}$ strongest (highest SNR) resolvable paths among the $\mathrm{L}$ resolvable ones [2]. We denote such hybrid schemes as SC/MRC receiver.

$\mathrm{SC} / \mathrm{MRC}$ receivers are expected to be more robust toward channel estimation errors since the weakest SNR paths (the ones that are the most exposed to these errors) are excluded from the combining process. Finally, SC/MRC was shown to approach the performance of MRC [2], while SC/EGC was shown to outperform in certain cases conventional post detection EGC since it is less sensitive to the "combining loss" of the very noisy (low SNR) paths [2].

In [2] and [3] Eng, Kong and Milstein present an error rate analysis of binary signal with GSC scheme (SC/MRC) over i.i.d. Rayleigh fading channels.

In this paper we show that, we are able to analyze the performance of GSC receiver over i.i.d. Nakagami and Rayleigh paths in terms of average combined SNR, and average error rate for arbitrary $\mathrm{M}$ and $\mathrm{L}$ over land mobile satellite communication channel.

Multicarrier direct sequence code-division multiple-access (MC DS-CDMA) is a multiple access scheme that intelligently combines the advantages of the orthogonal frequency division multiplexing (OFDM) and DS-CDMA schemes. It has the narrow band interference rejection capability and performance improvement (obtained via the RAKE receiver) of DS-CDMA systems. It also has the ability to send high data rates over hostile, frequency selective fading channels while maintaining high spectral efficiency via the OFDM scheme [4].

The transmitter of both multitone DS-CDMA and orthogonal MC DS-CDMA spreads the serial-to-parallel converted data streams using a given spreading code. In the multitone DS-CDMA system, the subcarrier frequencies are chosen to be orthogonal 
harmonics of each other with minimum frequency separation among them before DS spreading [5].

If Ts represents the symbol duration of the multitone DS-CDMA signal, then the spacing $(\Delta)$ between two adjacent subcarrier frequencies becomes $\left(\Delta=1 / \mathrm{T}_{\mathrm{s}}\right)$, and hence, the subcarrier frequencies take the values of $\left(f_{0}+i / T_{S}\right)$ for $i=0,1,2 \ldots, U-1$, where $\mathrm{f}_{0}$ is the fundamental frequency and $\mathrm{U}$ is the number of subcarrier frequencies. By contrast, in an orthogonal MC DS-CDMA system, the subcarrier frequencies are chosen to satisfy the orthogonality condition with the minimum possible frequency separation after DS spreading [4].

If $\mathrm{T}_{\mathrm{C}}$ is the chip duration of the $\mathrm{DS}$ spreading codes and the subcarrier frequencies take the values of $\left(\mathrm{f}_{0}+\mathrm{i} / \mathrm{T}_{\mathrm{C}}\right)$ for $\mathrm{i}=0,1,2 \ldots ., \mathrm{U}-1$ which implies that the spacing $(\Delta)$ between any two adjacent subcarrier frequencies becomes $\left(\Delta=1 / \mathrm{T}_{\mathrm{C}}\right)$. Let $\left(\mathrm{N}_{\mathrm{e}}=\mathrm{T}_{\mathrm{S}} / \mathrm{T}_{\mathrm{C}}\right)$ be the spreading gain of the DS-spread subcarrier signals, the orthogonality condition of both MC systems concerning the subcarrier frequencies can be expressed as $\left(\mathrm{f}_{0}+\mathrm{i} \Delta\right)$, $\mathrm{i}$ $=0,1, \ldots, \mathrm{U}-1$ and $\Delta=\lambda / \mathrm{T}_{\mathrm{S}}$ where $(\lambda=1,2, \ldots)$ is referred to the normalized spacing between two adjacent sub-carriers. If $\lambda=1$, the system becomes Multitone DS-CDMA, and if $\lambda=\mathrm{N}_{\mathrm{e}}$, the system becomes Orthogonal MC DS-CDMA [6].

In this paper, the performance of both Multitone DS-CDMA and Orthogonal MC DS-CDMA are evaluated over a land mobile satellite channel using Abdi model [7]. This model has the advantages of having simple, closed form and mathematically tractable expressions for the fundamental channel statistics. The performance evaluation is considered by using generalized selection combining (GSC(M,L)) that combines strongest $M$ diversity paths from total $L$ available paths where $M \leq L$ ( If $M=1$, it will be $\mathrm{SDC}$. If $\mathrm{M}=\mathrm{L}$, it will be $\mathrm{MRC}$ [8] ), at the receiver. The remainder of this paper is organized as follows. In the next section, the MC DS-CDMA system is described in detail. In section III, the statistics of the decision variables are analyzed. In section IV, the average probability of error is derived for the MC DS-CDMA system, and then the comparison numerical results are described in section V. Finally, the conclusion is presented in section VI.

\section{Multicarrier DS-CDMA System}

\section{A. Transmitted signal}

The transmitter schematic of the $\mathrm{k}^{\text {th }}$ user for the MC DS-CDMA system is shown in Fig.1. [5]. At the transmitter side, the incoming data stream having bit duration of $T_{b}$ is serial-to-parallel converted to U parallel sub-streams. The new bit duration of each substream or the symbol duration is $\mathrm{T}_{\mathrm{S}}=\mathrm{U} \mathrm{T}_{\mathrm{b}}$. After serial-to-parallel conversion, the $\mathrm{u}^{\text {th }}$ sub-stream modulates a sub-carrier frequency $f_{u, u} u=1,2, . ., \mathrm{U}$. The $\mathrm{U}$ sub-streams are then added together before spreading the composite signal with the DS-CDMA spreading code of the $\mathrm{k}^{\text {th }}$ user $\mathrm{c}_{\mathrm{k}}(\mathrm{t})$. Let $\mathrm{T}_{\mathrm{c}}$ be the chip duration of $\mathrm{c}_{\mathrm{k}}(\mathrm{t})$. The spreading 
gain of the DS-CDMA signal on each sub-carrier is given by $\mathrm{N}_{\mathrm{e}}=\mathrm{T}_{\mathrm{S}} / \mathrm{T}_{\mathrm{C}}$. The transmitted signal of the $\mathrm{k}^{\text {th }}$ user is given by

$$
S_{K}(t)=\sum_{u=1}^{U} \sqrt{2 P} b_{k u}(t) \cos \left(2 \pi f_{u} t+\phi_{k u}\right) C_{k}(t)=\sum_{u=1}^{U} S_{k u}(t)
$$

Where $P$ is the power per sub-carrier, $\Phi_{\text {ku }}$ is the phase angle introduced by the carrier $f_{u}$, $b_{k u}(\mathrm{t})$ is the waveform of the $\mathrm{u}^{\text {th }}$ sub-stream of the $\mathrm{k}^{\text {th }}$ user, given by

$b_{k u}(t)=\sum_{i=-\infty}^{\infty} b_{k u}(i) P_{T_{s}}\left(t-i T_{s}\right)$

Which consists of a sequence of mutually independent rectangular pulses of duration $T_{S}$ with amplitude $b_{k u}(i)= \pm 1$ with equal probability. The spreading sequence $c_{k}(t)$ is given by

$$
C_{k}(t)=\sum_{j=-\infty}^{\infty} C_{k}(j) P_{T_{c}}\left(t-j T_{c}\right)
$$

Which denotes the signature sequence waveform of the $\mathrm{k}^{\text {th }}$ user, where $c_{k}(j)$ assumes values of \pm 1 with equal probability, while $P_{T c}(t)$ is the rectangular chip waveform, defined over the interval $\left[0, \mathrm{~T}_{\mathrm{C}}\right)$. The composite signal, which is finally transmitted, is given by

$$
S(t)=\sum_{k=1}^{K} S_{k}(t)=\sum_{k=1}^{K} \sum_{u=1}^{U} S_{k u}(t)
$$

$\mathrm{n}$ order to have a convenient reference, we consider a single carrier DS-CDMA system occupying the same bandwidth $\mathrm{W}_{\mathrm{S}}$ as the MC DS-CDMA system and its spreading gain be $\mathrm{N}_{1}$. So, $\mathrm{N}_{\mathrm{e}}=\mathrm{UN}_{1}-(\mathrm{U}-1) \lambda / 2$ [6], this relation shows that as $\lambda$ increase, the spreading gain $\mathrm{N}_{\mathrm{e}}$ decrease, at the same bandwidth and subcarrier $\mathrm{U}$. Therefore, the spreading gain of multitone DS-CDMA $(\lambda=1)$ is higher than orthogonal MC DS-CDMA $\left(\lambda=\mathrm{N}_{\mathrm{e}}\right)$.

\section{B. Received Signal}

We assume that the channel between $\mathrm{k}^{\text {th }}$ user transmitter and the corresponding receiver is land mobile satellite channel (LMSC). This channel can be represented using Abdi model [7], which has a simple channel's equations form compared with Loo's model [9]. The total received signal in LMSC is given by $\mathrm{r} \exp (\mathrm{j} \Phi)=\mathrm{z} \exp (\mathrm{j} \theta)+\mathrm{w} \exp (\mathrm{j} \varphi)$

Where $\mathrm{r}, \mathrm{z}$ and $\mathrm{w}$ denote the amplitudes of the composite received signal, the LOS signal, and the multipath signal, respectively. While $\Phi, \theta$ and $\varphi$ denote the phases of these signals, respectively. The phase's $\theta$ and $\varphi$ are uniformly distributed between 0 and 
$2 \pi, \mathrm{z}$ is Nakagami distributed, and $\mathrm{w}$ is a Rayleigh distributed. The pdf of the received signal envelope $\mathrm{p}(\mathrm{r})$ is given by [7]

$$
p(r)=\left(\frac{2 b_{0} m}{2 b_{0} m+\Omega}\right)^{m} \frac{r}{b_{0}} \exp \left(-\frac{r^{2}}{2 b_{0}}\right){ }_{1} F_{1}\left(m, 1, \frac{\Omega r^{2}}{2 b_{0}\left(2 b_{0} m+\Omega\right)}\right), \quad r \geq 0
$$

here $b_{0}$ is average scattered power due to multipath component, $m$ is the Nakagami parameter $\mathrm{m}=\mathrm{E}^{2}\left[\mathrm{z}^{2}\right] / \operatorname{Var}\left[\mathrm{z}^{2}\right] \geq 0, \Omega=\mathrm{E}\left[\mathrm{z}^{2}\right]$ is the average power of the LOS component, and ${ }_{1} \mathrm{~F}_{1}(., .,$.$) is the confluent hyper-geometric function. It has been shown$ [7] that $r$ is Rayleigh distributed for small values of $m(m \approx 0)$ and Rice distributed for large values of $m(m \approx \infty)$ i.e.

$$
p(r)= \begin{cases}\frac{r}{b_{0}} \exp \left(-\frac{r^{2}}{2 b_{0}}\right), & m=0 \\ \frac{r}{b_{0}} \exp \left(-\frac{\left(r^{2}+\Omega\right)}{2 b_{0}}\right) I_{0}\left(\frac{\sqrt{\Omega} r}{b_{0}}\right), & m=\infty\end{cases}
$$

here $\mathrm{I}_{0}($.$) is the modified Bessel function of zeros order. If the transmitted signal of user$ $\mathrm{k}$ with U sub-carriers, given by (1), is passed through this channel, so, the channel complex low-pass equivalent impulse response will be given by [6]

$$
h_{k u}(t)=\sum_{l_{P}=0}^{L_{P}-1} \alpha_{u l_{P}}^{(k)} \delta\left(t-\tau_{k l_{P}}\right) \exp \left(-j \theta_{u l_{P}}^{(k)}\right)
$$

here $l_{p}$ is the channel (resolvable path) index, and $\alpha_{u l_{p}}^{(k)}$ is the fading amplitude for the $l_{p}^{\text {th }}$ channel of the $\mathrm{u}^{\text {th }}$ subcarrier of $\mathrm{k}^{\text {th }}$ user, $\tau_{k l_{p}}$ is the delay of the $l_{p}^{\text {th }}$ channel of $\mathrm{k}^{\text {th }}$ user, $\theta_{u l_{p}}^{(k)}$ is the phase-shift of the $l_{p}^{\text {th }}$ channel of the $\mathrm{u}^{\text {th }}$ subcarrier of $\mathrm{k}^{\text {th }}$ user, and $L_{p}$ is the total number of the resolvable paths. The fading amplitudes $\alpha_{u l_{p}}^{(k)}$ are assumed to be statistically independent and identical distributed (i.i.d) random variables whose pdf is given in (6), and the phase-shifts $\theta_{u l_{p}}^{(k)}$ are assumed to be (i.i.d.) random variables uniformly distributed in the interval $[0,2 \pi)$. The transmitted signal is also corrupted by an additive white Gaussian noise (AWGN) with zero mean and double-sided power spectral density of $\mathrm{N}_{0} / 2$. The AWGN is assumed to be statistically independent from path to another path and independent of the fading amplitudes $\alpha_{u l_{p}}^{(k)}$. Assuming slowfading channel, so, the sets of $\alpha_{u l_{P}}^{(k)}, \theta_{u l_{P}}^{(k)}$ and $\tau_{k l_{P}}$ are all constant over a symbol interval. Assume also that the multipath intensity profile (MIP) of the channel has an exponential form, so, $\Omega_{u l_{p}}^{(k)}=E\left[\left(\alpha_{u l_{p}}^{(k)}\right)^{2}\right]$ is the average power of the fade amplitude and given by [6]

$$
\Omega_{u l_{p}}^{(k)}=E\left[\left(\alpha_{u l_{p}}^{(k)}\right)^{2}\right]=\Omega_{u 0}^{(k)} \exp \left(-\eta l_{p}\right), \quad \eta \geq 0
$$

Where $\Omega_{u 0}^{(k)}$ is the average signal strength corresponding to the first resolvable path and $\eta$ is the rate of average power decay. Let $T_{m}$ be the maximum delay spread of the 
communication channel. Then, the number of resolvable paths $L_{p}$ associated with the MC DS-CDMA signal is given by [6]

$L_{p}=\left\lfloor T_{m} / T_{c}\right\rfloor+1$

here $\lfloor x\rfloor$ represents the largest integer not exceeding $x$. The number of resolvable paths $L_{1}$ of the corresponding single-carrier DS-CDMA signal with chip duration $\mathrm{T}_{\mathrm{C} 1}$ is given by [6]

$L_{1}=\left\lfloor T_{m} / T_{c 1}\right\rfloor+1$

Also from [6], the relation between $L_{p}$ and $L_{l}$ is given by

$L_{p} \approx\left\lfloor\frac{2 N_{e}(L-1)}{2 N_{e}+(U-1) \lambda}\right\rfloor+1$

\section{Receiver model}

Consider the correlator-based RAKE receiver in conjunction with generalized selection combining (GSC) as shown in Fig.2 and Fig.3. The composite received signal after passing through the proposed channel, can be expressed as

$$
r(t)=\sum_{k=1}^{K} \sum_{u=1}^{U} \sum_{l_{p}=0}^{L_{p}-1} \sqrt{2 p} \alpha_{u l_{p}}^{(k)} b_{k u}\left(t-\tau_{k l_{p}}\right) c_{k}\left(t-\tau_{k k_{p}}\right) \cos \left(2 \pi f_{u} t+\varphi_{u l_{p}}^{(k)}\right)+n(t)
$$

Where $\varphi_{u l_{p}}^{(k)}=\phi_{k u}-\theta_{u l_{p}}^{(k)}-2 \pi f_{u} \tau_{k l_{p}}$, is the total received signal phase which is assumed to be an i.i.d. random variable having a uniform distribution in $[0,2 \pi)$. Without loss of generality, we consider the receiver for the $1^{\text {st }}$ user (user of interest) i.e. $\mathrm{k}=1$.

Assume that the receiver uses diversity combining paths $\left(L \leq L_{p}\right)$ which represents the number of diversity branches that will be ordered statistics and choose strongest $M$ branches of them as a GSC(M,L) diversity technique used by the receiver, we also assume perfect channel estimation at the receiver, i.e. the receiver can correctly estimate $\left\{\alpha_{u L}^{(1)}\right\}$ and $\left\{\varphi_{u L}^{(1)}\right\}$ it is also capable for acquiring perfect time domain synchronization with each path of the reference signal.

Let $\left\{\alpha_{u 1: L}^{(1)} \geq \alpha_{u 2: L}^{(1)} \geq \ldots . . \geq \alpha_{u L: L}^{(1)} \geq 0\right\}$ be the order statistics obtained by arranging the $\left\{\alpha_{u l}^{(1)}\right\}_{l=1}^{L}$ in decreasing order of magnitude and since the $\left\{\alpha_{u l}^{(1)}\right\}_{l=1}^{L}$ are i.i.d., the joint pdf

$P_{\alpha_{1: L}, \ldots, \alpha_{M: L}}\left(\alpha_{1: L}, \ldots \ldots, \alpha_{M: L}\right)$ of the $\left\{\alpha_{u l}^{(1)}\right\}_{l=1}^{M}(\mathrm{M} \leq \mathrm{L})$ is given by [10] :

$P_{\alpha_{1: L}, \ldots, \alpha_{M: L}}\left(\alpha_{1: L}, \ldots . ., \alpha_{M: L}\right)=M !\left(\begin{array}{c}L \\ M\end{array}\right)\left[p_{\alpha}\left(\alpha_{M: L}\right)\right]^{L-M} \prod_{l=1}^{M} P_{\alpha}\left(\alpha_{l: L}\right)$ 
Where $p_{\alpha}\left(\alpha_{M: L}\right)$ is the pdf of the $\left\{\alpha_{u l}^{(1)}\right\}_{l=1}^{L}$ given in (6) and $P_{\alpha}\left(\alpha_{l: L}\right)$ is the CDF of $\left\{\alpha_{u l}^{(1)}\right\}_{l=1}^{L}$.

The decision variable at the combiner output for the $v^{\text {th }}$ sub-carrier is denoted as $Z_{v}$

$$
Z_{v}=\sum_{l=0}^{M-1} Z_{v l} \quad, \quad v=1,2, \ldots \ldots, \quad U
$$

here

$$
Z_{v l}=\int_{\tau_{1 l}}^{T_{s}+\tau_{1 l}} r(t) \alpha_{v l}^{(1)} c_{1}\left(t-\tau_{1 l}\right) \cos \left(2 \pi f_{v} t+\varphi_{v l}^{(1)}\right) d t
$$

The signal is then decoded by making hard decision on $Z_{v}$, i.e. the current data bit is decided to be 0 or 1 depending on, if $Z_{v}$ is higher than zero or not. Finally, the $U$ parallel-decoded sub-streams are parallel-to-serial converted and the serial stream is output which represents the recovered data.

\section{Decision Variable Statistics}

Without loss of generality, in the following analysis we assume that $\left(\tau_{1 l}=0\right)$ for simplicity, and we refer to the current bit to be decoded as $b_{u}[0]$, the previous bit as $b_{u}[-$ 1] and the next bit as $b_{u}[1]$. We also assume that the spreading sequences $c_{k j}$ are random. Then by substituting (13) into (16), it can be shown that $\mathrm{Z}_{\mathrm{vl}}$ can be written as $Z_{v l}=D_{v l}+N_{v l}+I_{1}^{(S)}+I_{2}^{(S)}+I_{1}^{(K)}+I_{2}^{(K)}$

Where $D_{v l}$ is the desired term derived by substituting (13) into (16) and setting k=1, $l_{p}=l$ and $u=v, N_{v l}$ is the noise term contributed by $n(t)$ of (13) and it has zero mean and variance $\left(\alpha{ }_{v l}^{(1)}\right)^{2} N_{0} / 2 E_{b}$, where $\mathrm{E}_{\mathrm{b}}=\mathrm{PT}_{\mathrm{S}}$ is the energy per bit, $I_{1}^{(S)}$ is the self-interference term that comes from setting $\mathrm{k}=1, u=v$, and all paths $l_{p}$ except $l_{p}=l, I_{2}^{(S)}$ is the selfinterference term that comes from setting $\mathrm{k}=1$ and all paths $l_{p}$ except $l_{p}=l$ and all subcarriers $u$ except $u=v, I_{1}^{(K)}$ is the multi-user interference term that comes from all paths $l_{p}$ and $u=v$ but $\mathrm{k}=2,3, \ldots, \mathrm{K}$, and $I_{2}^{(K)}$ is the multi-user interference term that comes from all paths $l_{p}$ and all sub-carriers $u$ except $u=v$ and $\mathrm{k}=2,3, \ldots, \mathrm{K}$, The correlator output $\mathrm{Z}_{\mathrm{vl}}$ of (17) can be approximated as a Gaussian random variable with normalized mean given by

$$
E\left[Z_{v l}\right]=D_{v l}=\left(\alpha_{v l}^{(1)}\right)^{2} b_{v}[0]
$$

and normalized variance given by 


$$
\begin{aligned}
\operatorname{Var}\left[Z_{v l}\right]= & \left\{\frac{\left(\alpha_{v l}^{(1)}\right)^{2} N_{0}}{2 E_{b}}+\sum_{\substack{l_{p}=0 \\
l_{p} \neq l}}^{L_{p}-1} \frac{\Omega_{u l_{p}}^{(1)}\left(\alpha_{v l}^{(1)}\right)^{2}}{3 N_{e}}+\sum_{\substack{u=1 \\
u \neq v l_{p} \neq 0}}^{U} \sum_{l_{p}-1}^{L_{p}-1} \frac{\Omega_{u l_{p}}^{(1)}\left(\alpha_{v l}^{(1)}\right)^{2} N_{e}(u-v)^{2} \lambda^{2}}{2 \pi^{2}}\left[1-\sin c\left(\frac{2 \pi(u-v) \lambda}{N_{e}}\right)\right]\right. \\
& \left.+\sum_{k=2}^{K} \sum_{l_{p}=0}^{L_{p}-1} \frac{\Omega_{u l_{p}}^{(k)}\left(\alpha_{v l}^{(1)}\right)^{2}}{3 N_{e}}+\sum_{k=2}^{K} \sum_{\substack{u=1 \\
u \neq v}}^{U} \sum_{l_{p}=0}^{L_{p}-1} \frac{\Omega_{u l_{p}}^{(k)}\left(\alpha_{v l}^{(1)}\right)^{2} N_{e}}{2 \pi^{2}(u-v)^{2} \lambda^{2}}\left[1-\operatorname{sinc}\left(\frac{2 \pi(u-v) \lambda}{N_{e}}\right)\right]\right\}
\end{aligned}
$$

So, $\operatorname{Var}\left[Z_{v l}\right]$ in Eq. (19) can be simplified as [6]

$\operatorname{Var}\left[Z_{v l}\right]=\left[\left(\frac{2 \Omega_{0} E_{b}}{N_{0}}\right)^{-1}+\frac{\left(K L_{p}-1\right)\left(1-\exp \left(\eta L_{P}\right)\right)}{L_{P}(1-\exp (\eta))}\left(\frac{1}{3 N_{e}}+(U-1) \bar{I}_{M}\right)\right] \Omega_{0}\left(\alpha_{v l}^{(1)}\right)^{2}$

Where

$$
\bar{I}_{M}=\frac{1}{U(U-1)} \sum_{\substack{u=1 \\ u=1 \\ u \neq v}}^{U} \frac{N_{e}}{2 \pi^{2}(u-v)^{2} \lambda^{2}}\left[1-\operatorname{sinc}\left(\frac{2 \pi(u-v) \lambda}{N_{e}}\right)\right]
$$

is the average of $I_{2}^{(S)}$ and/or $I_{2}^{(K)} \quad$ [6]. For simplicity, we assume that all the sub-carriers signals of the different users obey the same multi-path intensity profile (MIP) distribution of the channel, i.e.

$$
\Omega_{u l_{p}}^{(K)}=\Omega_{l_{P}}=\Omega_{0} \exp \left(\eta l_{P}\right), \quad \forall k=1,2, \ldots, K \text { and } u=1,2, \ldots, U \text { andl } l_{P}=0,1, \ldots L_{P}-1
$$

Where $\Omega_{0}$ is the average signal power corresponding to the first resolvable path, and $\eta$ is the rate of average power decay.

\section{Performance Analysis}

As we have discussed in the above section, the decision variable, $\mathrm{Z}_{\mathrm{vl}}$ for $\mathrm{v}=1,2, . ., \mathrm{U}$ and for the $l^{\text {th }}$ branch of the strongest M-branches that were chosen from totally Lbranches as a GSC(M,L) diversity technique used at receiver, can be approximated as a Gaussian random variable having a normalized mean given by (18) and a normalized variance given by (19). Therefore, the BER using BPSK modulation can be expressed as

$$
P_{b}\left(E \mid \gamma_{l}\right)=Q\left(\sqrt{\frac{\left(E\left[Z_{v l}\right]\right)^{2}}{\operatorname{Var}\left[Z_{v l}\right]}}\right)=Q\left(\sqrt{2 \gamma_{l}}\right)
$$

Where $\gamma_{1}$ is the signal to noise ratio of the $l^{\text {th }}$ channel and given by

$$
\begin{aligned}
& \gamma_{l}=\gamma_{c} \frac{\left(\alpha_{v l}^{(1)}\right)^{2}}{\Omega_{0}} \\
& \gamma_{c}=\left[\left(\frac{\Omega_{0} E_{b}}{N_{0}}\right)^{-1}+\frac{2\left(K L_{P}-1\right)\left(1-\exp \left(-\eta L_{P}\right)\right)}{L_{P}(1-\exp (-\eta))}\left(\frac{1}{3 N_{e}}+(U-1) \bar{I}_{M}\right)\right]^{-1}
\end{aligned}
$$


By applying the alternative representation of the Gaussian $Q$-function [11] on the conditional BER of (22) we find that

$$
P_{b}\left(E \mid \gamma_{l}\right)=\frac{1}{\pi} \int_{0}^{\pi / 2} \exp \left(-\frac{\gamma_{l}}{\sin ^{2} \theta}\right) d \theta
$$

This form of the conditional BER is desirable, since we can first independently average over the probability density function (pdf) of the strongest (M) from (L) independent squared path gains in the case of generalized selection diversity combining GSC(M,L) which denoted by $P_{G S C}(\alpha)$ in case of our channel model (LMSC) Abdi [7], and then perform the integral over $\theta$. The probability density function $P_{G S C}(\alpha)$ of Abdi channel model is obtained by applying the pdf and the CDF of Abdi channel model in the pdf formula of GSC(M,L) given by (14), so we find that :

$$
\begin{aligned}
P_{G S C}(\alpha)=M !\left(\begin{array}{l}
L \\
M
\end{array}\right)\left[\left(\frac{2 b_{0} m}{2 b_{0} m+\Omega}\right)^{m} \frac{\alpha}{b_{0}} \exp \left(-\frac{\alpha^{2}}{2 b_{0}}\right){ }_{1} F_{1}\left[m, 1, \frac{\Omega \alpha^{2}}{2 b_{0}\left(2 b_{0} m+\Omega\right)}\right]\right]^{L-M} \\
\prod_{l=1}^{M} \int_{0}^{\alpha}\left[\left(\frac{2 b_{0}}{2 b_{0} m+\Omega}\right)^{m} \frac{r}{b_{0}} \exp \left(-\frac{r^{2}}{2 b_{0}}\right){ }_{1} F_{1}\left[m, 1, \frac{\Omega r^{2}}{2 b_{0}\left(2 b_{0} m+\Omega\right)}\right]\right] d r
\end{aligned}
$$

So the average bit error rate $\bar{P}_{e}$ could be obtained

$$
\bar{P}_{e}=\int_{0}^{\infty} P_{b}(\gamma / \alpha) P_{G S C}(\alpha) d \alpha
$$

By substitute of (25) \& (26) into (27) we find that

$$
\begin{aligned}
\bar{P}_{e}=M !\left(\frac{1}{\pi}\right)\left(\begin{array}{l}
L \\
M
\end{array}\right)\left(\frac{2 b_{0} m}{2 b_{0} m+\Omega}\right)^{m L} \int_{0}^{\infty} \int_{0}^{\pi / 2} \int_{0}^{\alpha} & \prod_{l=0}^{M-1} \exp \left(-\frac{\gamma_{l}}{\sin ^{2} \theta}\right)\left[\frac{\alpha}{b_{0}} \exp \left(\frac{\alpha^{2}}{2 b_{0}}\right){ }_{1} F_{1}\left[m, \frac{\Omega \alpha^{2}}{2 b_{0}\left(2 b_{0} m+\Omega\right)}\right]\right]^{L-M} \\
& {\left[\frac{r}{b_{0}} \exp \left(-\frac{r^{2}}{2 b_{0}}\right){ }_{1} F_{1}\left[m, \frac{\Omega r^{2}}{2 b_{0}\left(2 b_{0} m+\Omega\right)}\right]\right]^{M} d r d \theta d \alpha }
\end{aligned}
$$

Note that in (25) we replace $\gamma_{l}$ by $\left\{\gamma_{c} \alpha^{2} / \Omega_{0}\right\}$, and the parameters $\mathrm{b}_{0}, \mathrm{~m}$ and $\Omega$ are mentioned in table 1 , for the light, average and heavy shadowing.

\section{Table 1: channel model parameters}

\begin{tabular}{|c|c|c|c|}
\hline & Average Shadowing & Light Shadowing & Heavy Shadowing \\
\hline $\mathrm{b}_{0}$ & 0.126 & 0.158 & 0.063 \\
\hline $\mathrm{m}$ & 10.1 & 19.4 & 0.739 \\
\hline$\Omega$ & 0.835 & 1.29 & $8.97 \times 10^{-4}$ \\
\hline
\end{tabular}

\section{Numerical Results}


The channel is specified in terms of a single-carrier DS-CDMA system that occupying the same bandwidth as the MC DS-CDMA system. The spreading gain and the number of resolvable paths of the corresponding single-carrier DS-CDMA are $N_{l}=128$ and $L_{1}$ $=32$ respectively. The MIP decay factor is $\eta=0.2$. The number of sub-carriers $U$ is 32 . The normalized sub-carrier spacing $\lambda$ is equal to 1 for MT DS-CDMA as mentioned above, but for the orthogonal MC DS-CDMA system, $\lambda$ is calculated as and 248 by using $\lambda=N_{e}$ in the relation $\left\{\mathrm{N}_{\mathrm{e}}=\mathrm{U} \mathrm{N} \mathrm{N}_{1}-(\mathrm{U}-1) \lambda / 2\right\}$ that described above. The number of resolvable paths $L_{P}$ for the MC DS-CDMA system is calculated using $N_{e}, U$ and $\lambda$ in (12).

Fig. 4 : Presents the effect of the normalized sub-carrier spacing $\lambda$ on the number of resolvable paths $L_{p}$ of MC DS-CDMA. We see that the number of resolvable paths $L_{p}$ decrease with increase in the sub-carrier spacing, this due to the decrease of the spreading gain $N_{e}$ with $\lambda$ increase. Also, as the number of sub-carriers increase, the number of resolvable paths becomes lower.

Fig. 5, 6 and 7 : Shows the average BER versus SNR for light, average and heavy shadowing and fading with number of diversity paths $\mathrm{L}=5$ and $\mathrm{M}$ varying from 1 (SDC) to 3 then to 5 (MRC) for both multitone DS-CDMA and orthogonal MC DS-CDMA. We see that the average BER decreases when the level of shadowing and fading decreases or when the bit energy increases, also as $M$ increase the system performance gets better, but system complexity becomes high .

Fig. 8, 9 and 10 : Shows the average BER versus the number of the simultaneously active users $\mathrm{K}$ at $\mathrm{E}_{\mathrm{b}} / \mathrm{N}_{\mathrm{o}}=15 \mathrm{~dB}$ for both multitone DS-CDMA and orthogonal MC DSCDMA at number of diversity paths $\mathrm{L}=5$ and $\mathrm{M}$ varying from 1 (SDC) to 3 then to 5 (MRC). This figures shows that, as $\mathrm{K}$ increase, the average BER increase also for all level of shadowing and vice verse and as $M$ changes from 1 to 5 , the performance improved. And it is found that the Orthogonal MC DS-CDMA system has better performance than the Multitone DS-CDMA for all the time.

\section{Conclusion}

As shown in Fig.4., if $\lambda$ is low, as in multitone DS-CDMA $(\lambda=1)$, then, a subcarrier signal will overlap with a high number sub-carrier signals of both the same user and with those of the interfering users which leads to high interference inflicted by the sub-carrier signals. On the other hand, given a total bandwidth and low value of $\lambda$, a high spreading gain can be maintained, which leads to the reduction of the multi-user interference. By contrast, if $\lambda$ is high, as in Orthogonal MC DS-CDMA $\left(\lambda=N_{e}\right)$, which mean that there exists low spectral overlap between the main lobes of the sub-carrier signals, then, the modulated sub-carrier signals benefit from a low interference inflicted by the sub-carrier signals of both the reference and the interfering users. However, in this case the spreading gain of each sub-carrier signal is low, which leads to the increase of the multi-user interference. Using diversity at the receiver improve the system 
performance, and the GSC(M,L) diversity technique matches between performance and complexity as it is changing from $\operatorname{SDC}($ at $\mathrm{M}=1)$ and $\mathrm{MRC}($ at $\mathrm{M}=\mathrm{L})$ diversity techniques. In general, the Orthogonal MC DS-CDMA system has better performance than the Multitone DS-CDMA.

\section{References}

[1] Richard D.J., Van Nee and Ramjee Prasad, "Spread-Spectrum path diversity in a shadowed

Rician fading land mobile satellite channel " ; IEEE Vech. Tech., Vol.42, May 1993, pp.131-

136.

[2] T. Eng, N. Kong and L.B Milstein, " Comparison of diversity combining techniques for Rayleigh-fading channels ", IEEE Trans. Comm. , vol. COM- 46, September 1998, PP. 1111.

[3] N. Kong, T. Eng and L.B Milstein, " A selection combining scheme for RAKE receivers ", in proc. IEEE Int. Conf. Univ. Personal Comm. (ICUPC' 95), Tokyo, November 1995, PP. 426-429.

[4] R.Prasad, S.Hara , " An overview of multi-carrier CDMA " ; IEEE Commun. Mag., Dec 1997, pp.126-133.

[5] L.vandendorpe, "Multitone spread spectrum multiple access communications system in a

multipath Rician fading channel, "IEEE Trans. Veh. Technol., vol. 44, 1995, pp. 327-337.

[6] Lie-Liang Yang, Lajos hanzo, " Performance of generalized MC DS-CDMA over Nakagami- $m$

fading channels ", IEEE Trans. on comm., vol. 50 , Jun. 2002, pp. 956-966.

[7] A. Abdi , H. Allen Barger, and M. Kaveh, " A simple Alternative to the lognormal model for

shadow fading in terrestrial and satellite channels ", Proc. IEEE Vech. Tech. Conf. , Atlantic

city, NJ.,2001,PP. 2058-2062.

[8] G. Deora, "Simulation and Mathematical Tools for Performance Analysis of LowComplexity Receivers ", Master thesis, January 2003, Blacksburg, Va. [9] C.Loo, "A statistical model for a Land Mobile Satellite Link ", IEEE Trans. Vech. Tech., Vol.

34, August 1985, pp.122-127.

[10] A. Papoulis, " Probability, Random variables and Stochastic processes ", $2^{\text {nd }}$ ed. , New York; McGrow-Hill , 1991. 
[11] M.-S. Alouini and A.J. Goldsmith, "A unified approach for calculating error rates of linearly

modulated signals over generalized fading channels ", IEEE Trans. comm., Vol. 47, Sept. 1999,

PP. 1324-1334.

[12] J.G. Proakis, "Digital communications", $3^{\text {rd }}$ ed., New York, McGraw-Hill, 1995.

[13] Gamal Mabrouk Abdel-Hamid, El-Sayed A. Gadallah. And Salah El-agooz," Analysis of 16-ary Quadrature Amplitude Modulation Direct-Sequence Spread Spectrum for Land Mobile Satellite Communication Channels ", $3^{\text {rd }}$ ICEENG Conference on Electrical Engineering, May 2002, 14-16, Military Technical CollegeEgypt.

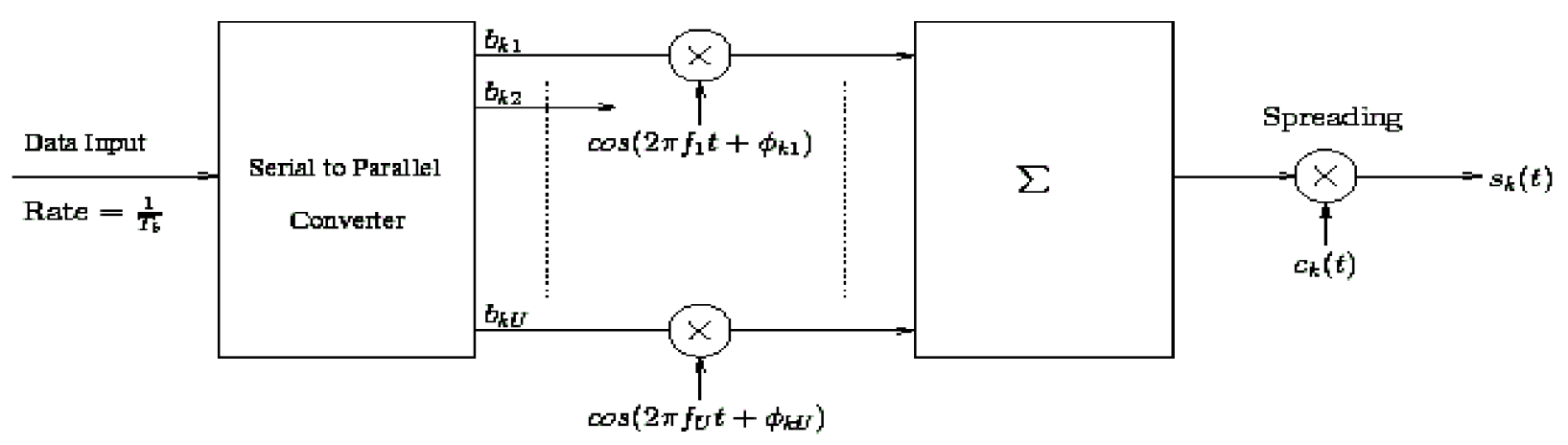

Fig. 1. Transmitter of $k^{\text {th }}$ user

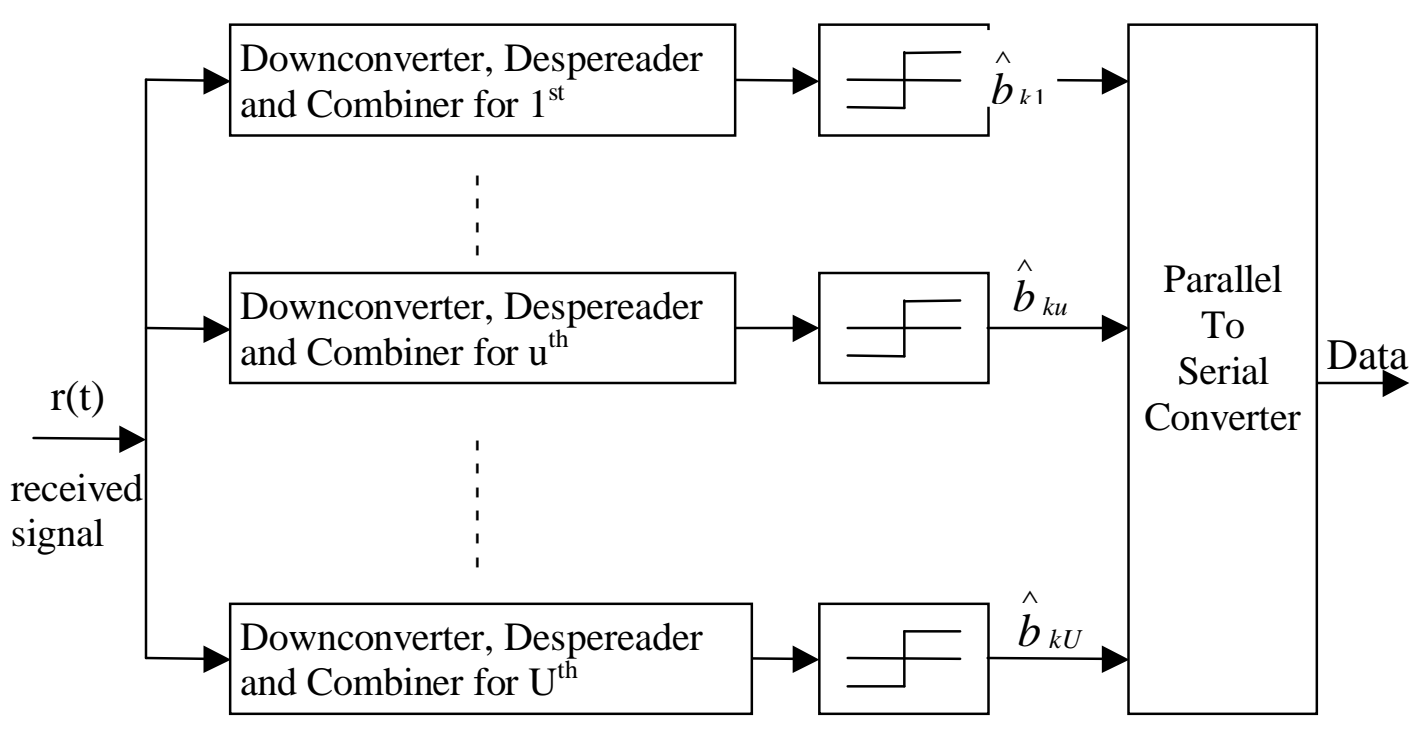


Fig. 2. Block diagram of the receiver for the $k^{\text {th }}$ user

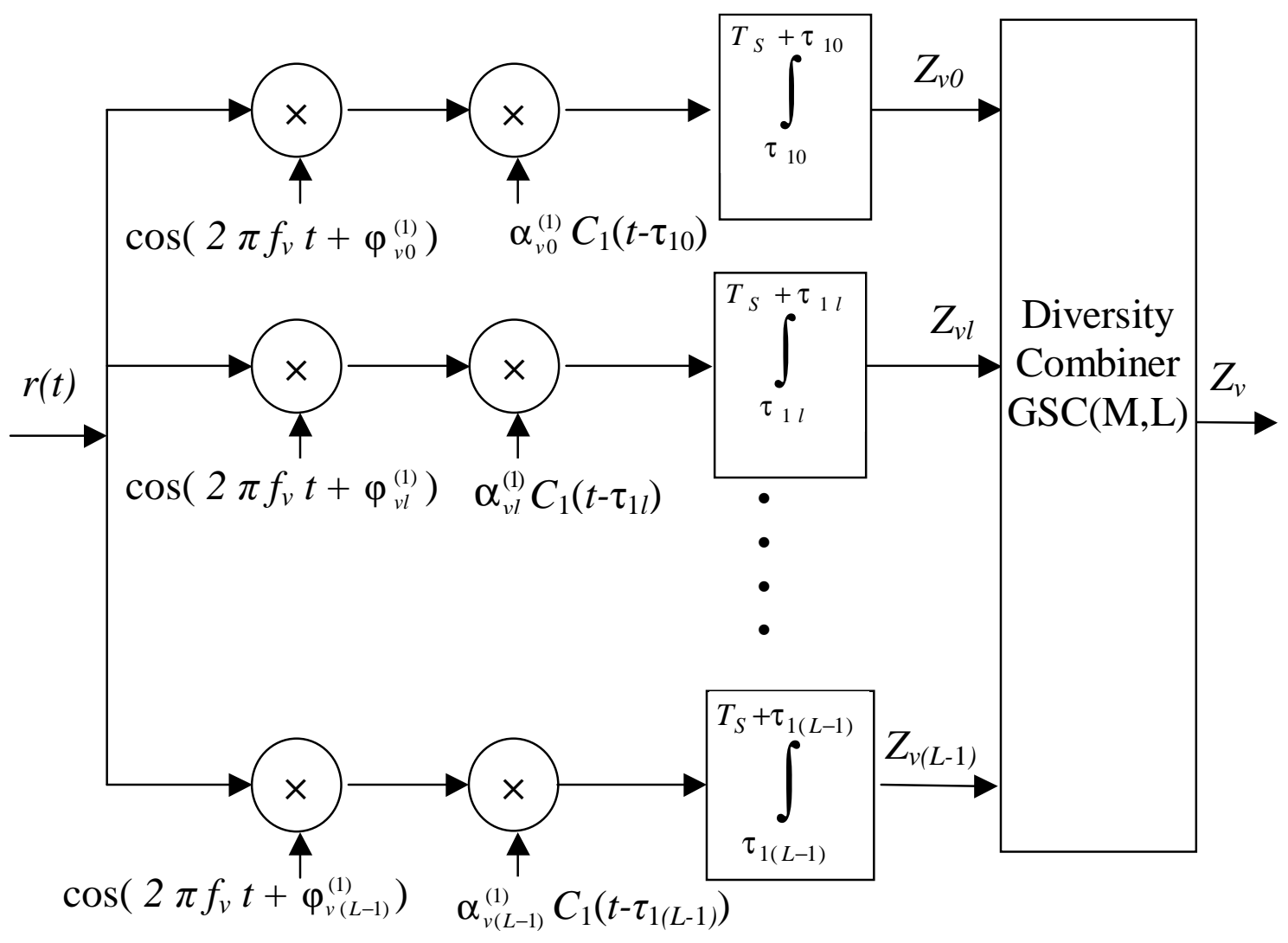

Fig.3. The down-converter, despreader and combiner for the $v^{\text {th }}$ sub-carrier of $1^{\text {st }}$ user $(k=1)$ 


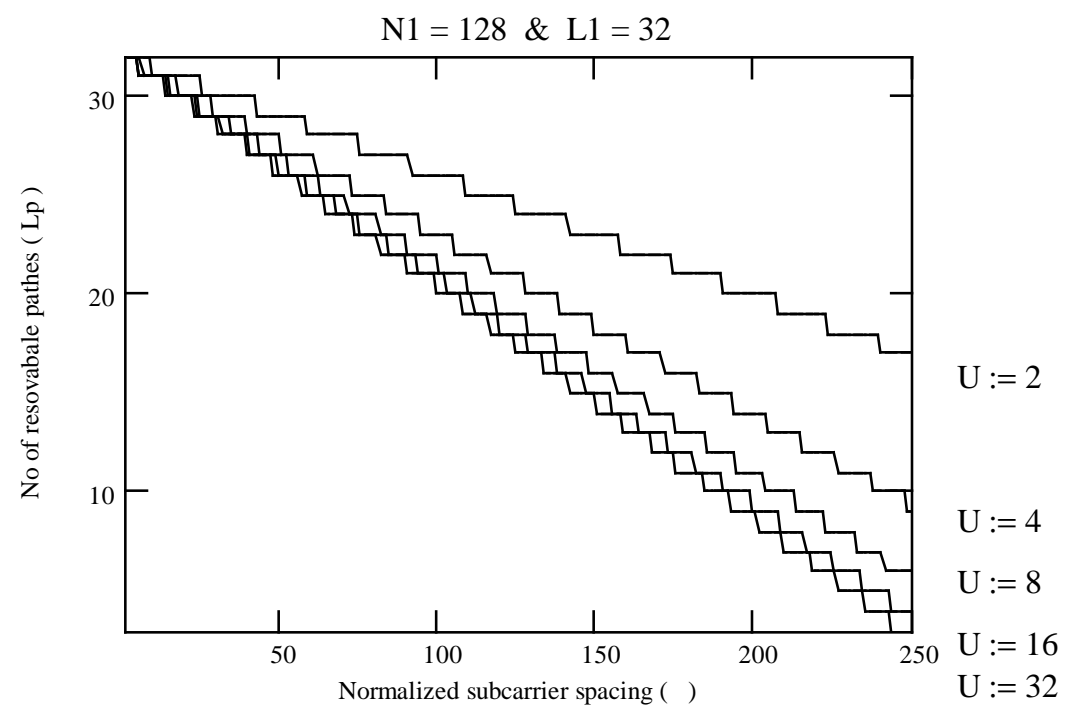

Fig.4. The effect of the normalized sub-carrier spacing $\lambda$ on the number of resolvable paths $L_{p}$

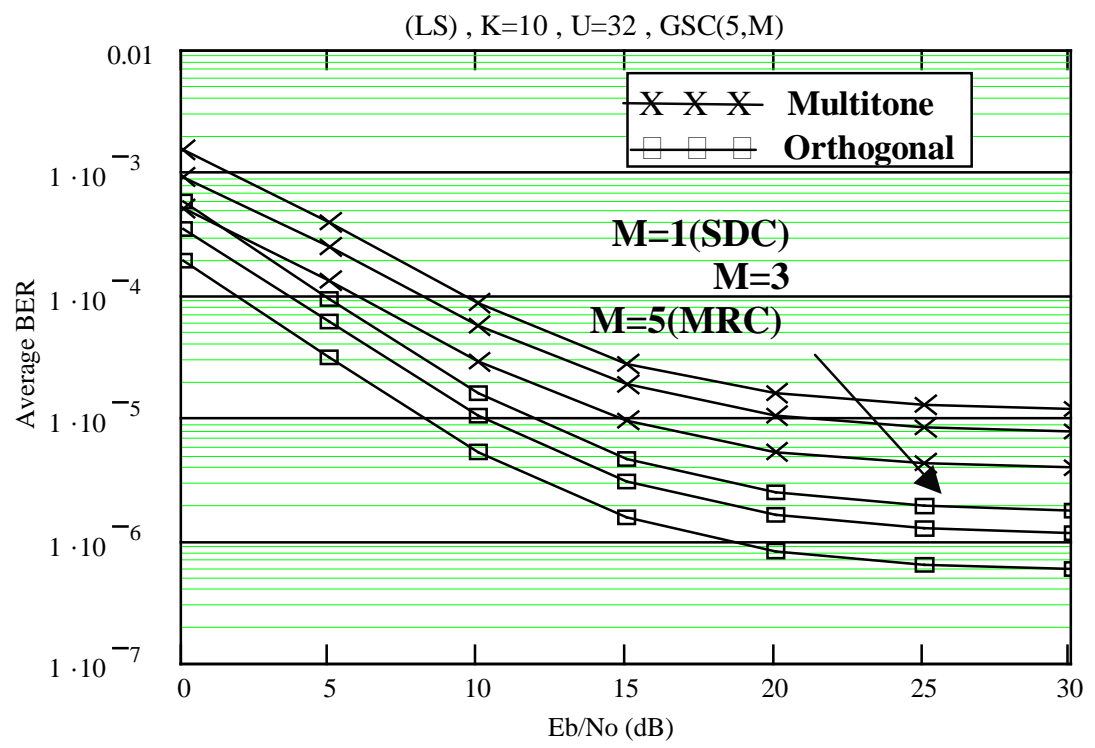

Fig. 5. Average BER against $\mathrm{E}_{\mathrm{b}} / \mathrm{N}_{0}$ in (dB) at light shadowing (LS) with $\mathrm{GSC}(\mathrm{M}, 5)$ and $M=1(S D C) \& M=3 \& M=5(M R C)$ 


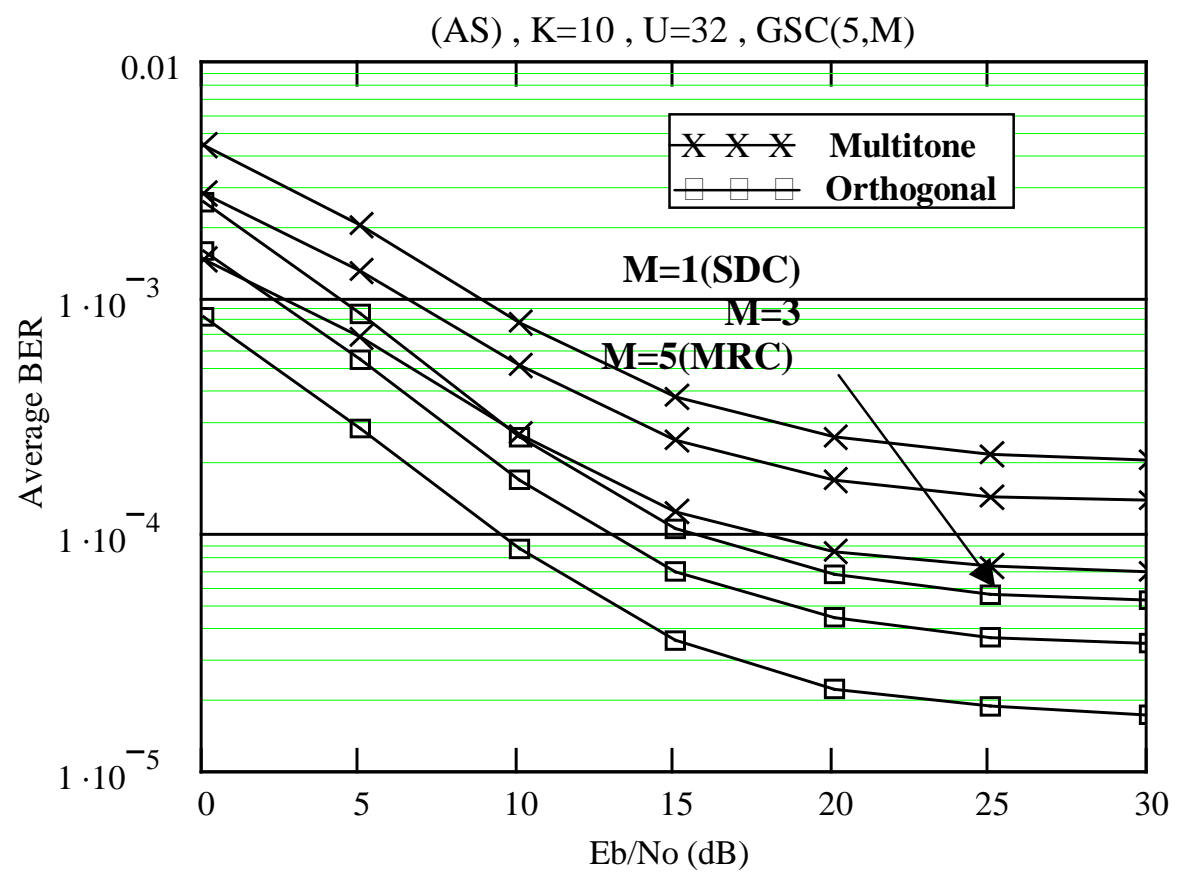

Fig. 6. Average BER against $\mathrm{E}_{\mathrm{b}} / \mathrm{N}_{0}$ in (dB) at average shadowing (AS) GSC(M,5) and $M=1(S D C) \& M=3 \& M=5(M R C)$

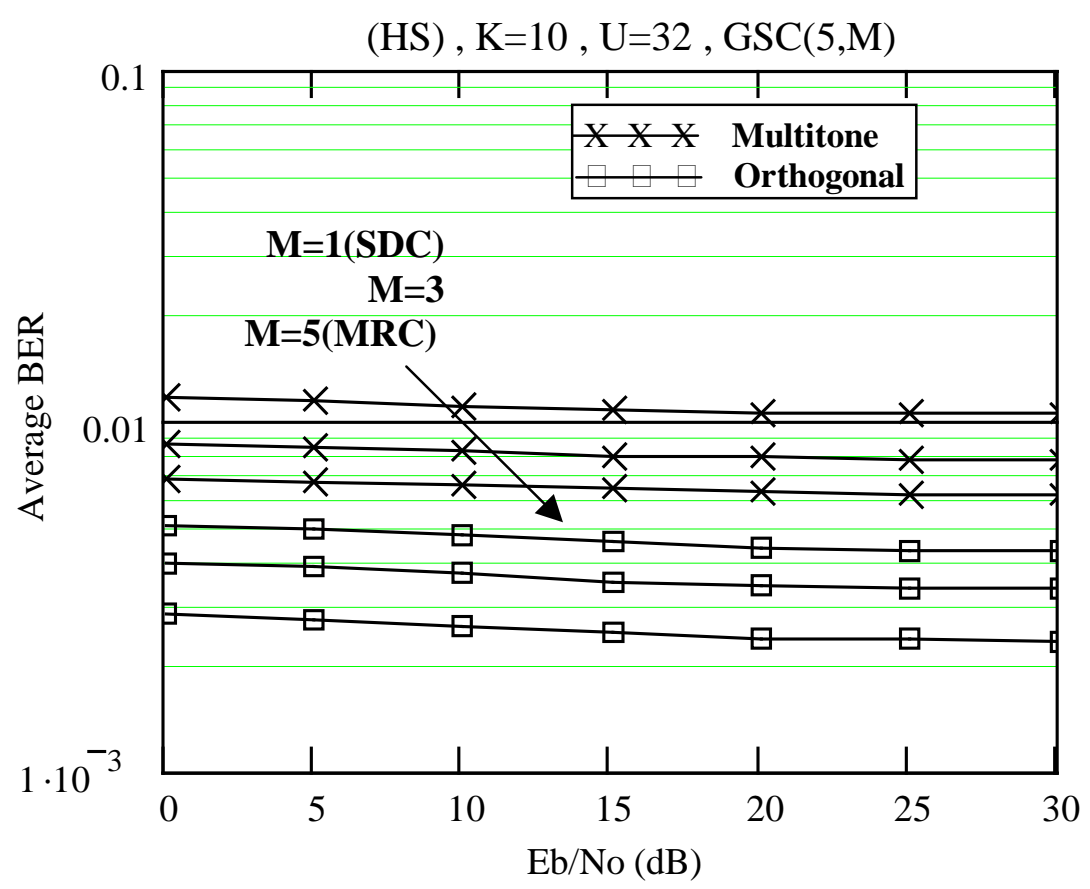


Fig. 7. Average BER against $E_{b} / N_{0}$ in (dB) at heavy shadowing (HS) with GSC $(M, 5)$ and $M=1(S D C) \& M=3 \& M=5(M R C)$

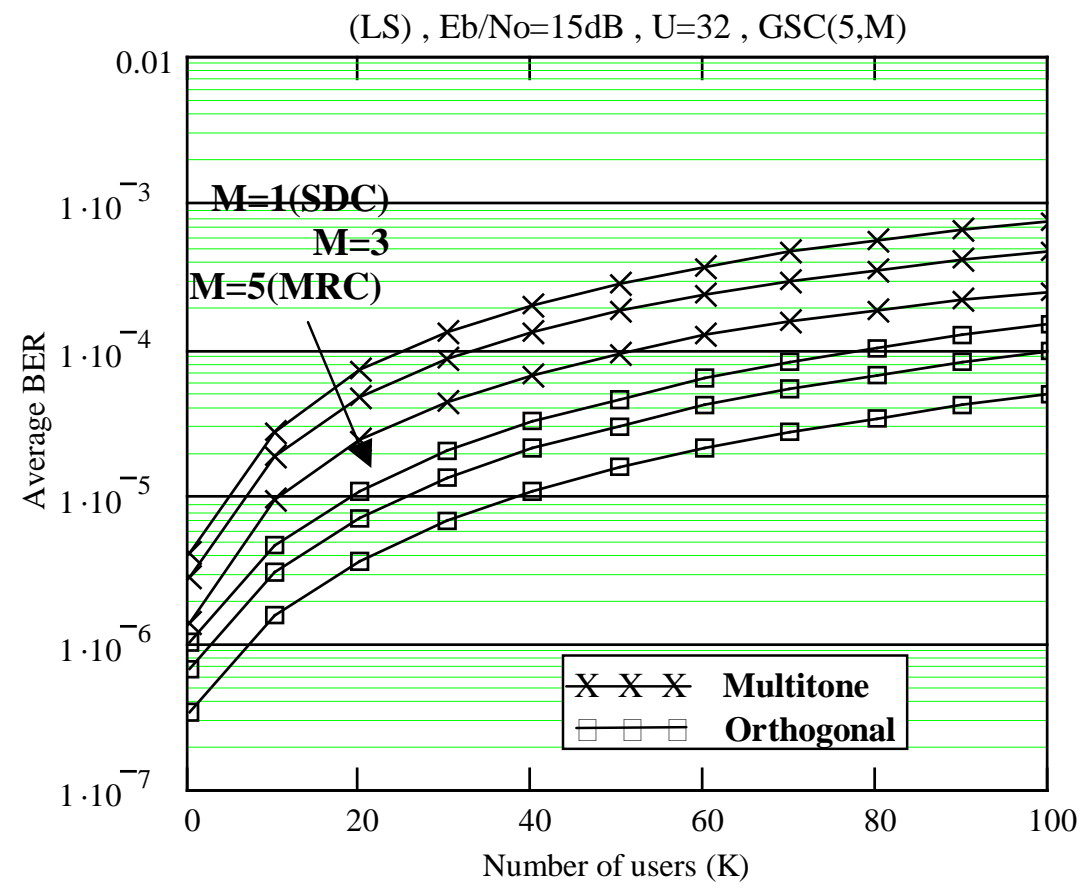

Fig. 8. Average BER against number of users (K) at (LS) \& $E_{b} / N_{0}=15 d B$ with GSC (M,5) and M=1(SDC) \& M=3 \& M=5(MRC)

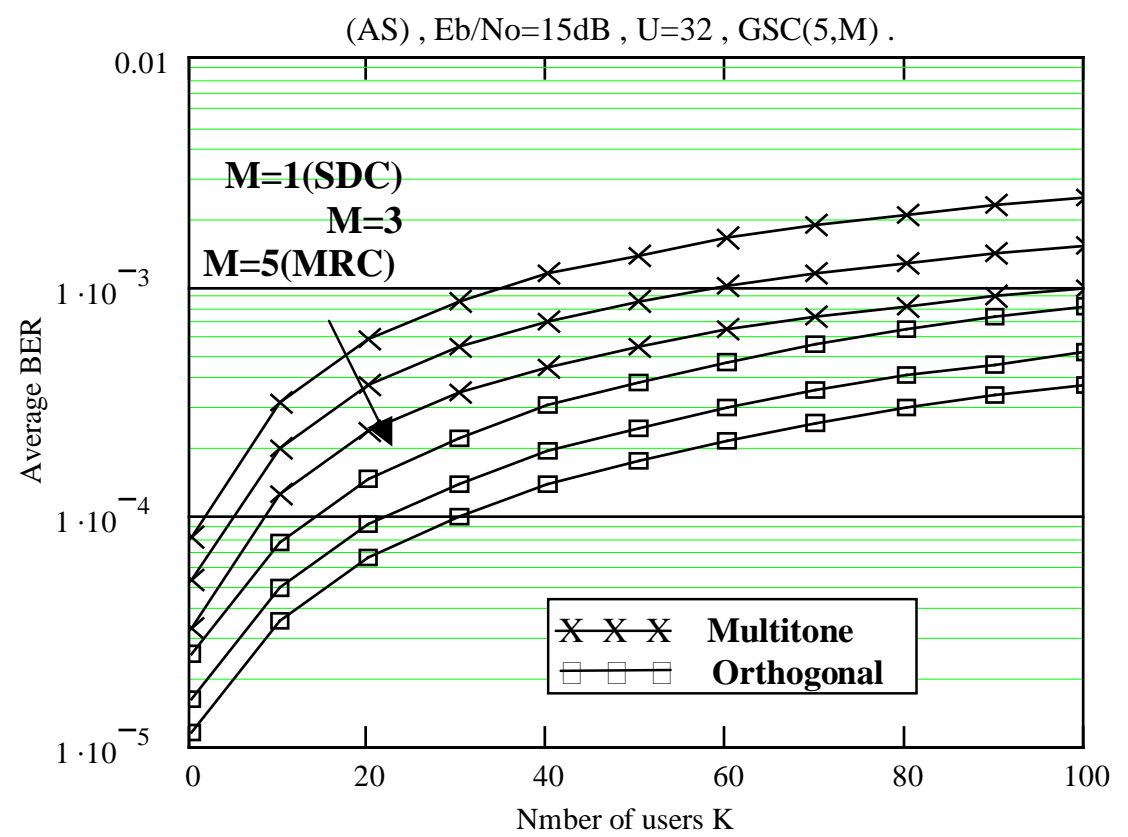


Fig. 9. Average $B E R$ against number of users (K) at (AS) \& $E_{b} / N_{0}=15 d B$ with GSC(M,5) and M=1(SDC) \& M=3 \& M=5(MRC)

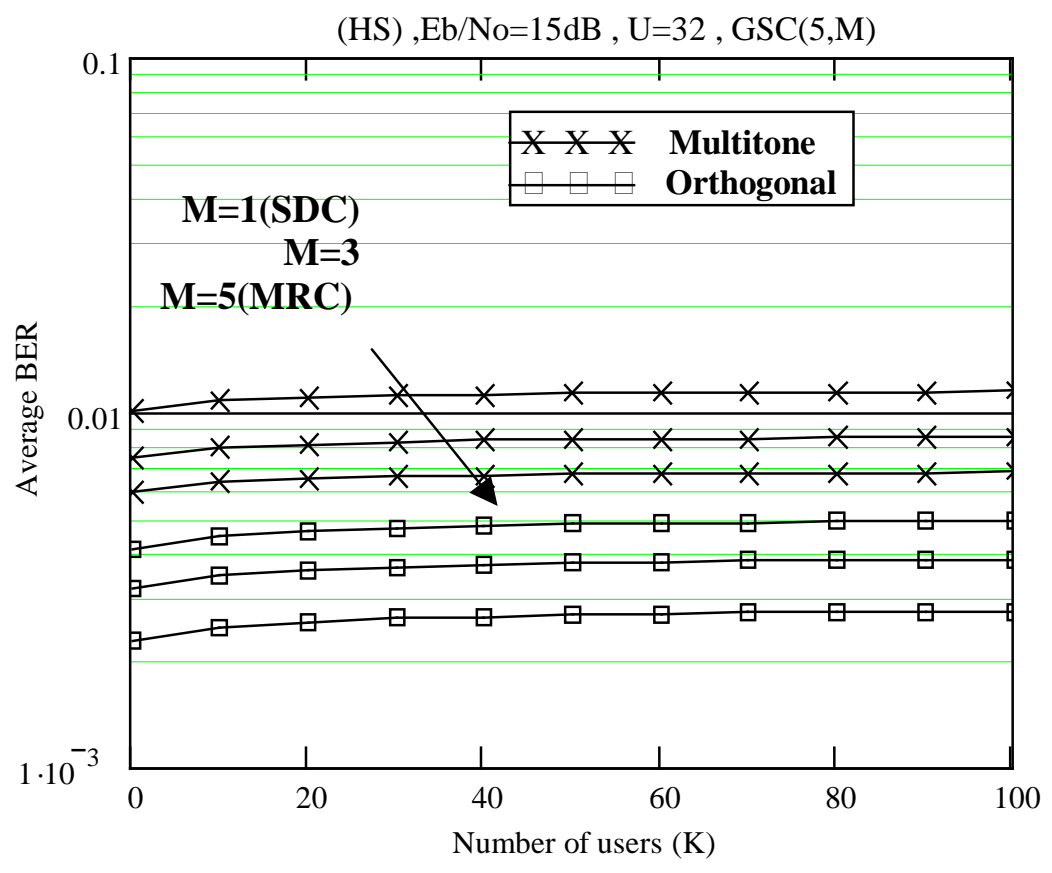

Fig. 10. Average BER against number of users (K) at (HS) \& $E_{b} / N_{0}=15 d B$ with GSC(M,5) and M=1(SDC) \& M=3 \& M=5(MRC) 